\title{
A Wearable Fall Detection System Based on LoRa LPWAN Technology
}

\author{
Elma Zanaj, Deivis Disha, Susanna Spinsante, Senior Member, IEEE, and Ennio Gambi, Senior Member, IEEE
}

\begin{abstract}
Several technological solutions now available in the market offer the possibility of increasing the independent life of people who by age or pathologies otherwise need assistance. In particular, internet-connected wearable solutions are of considerable interest, as they allow continuous monitoring of the user. However, their use poses different challenges, from the real usability of a device that must still be worn to the performance achievable in terms of radio connectivity and battery life. The acceptability of a technology solution, by a user who would still benefit from its use, is in fact often conditioned by practical problems that impact the person's normal lifestyle. The technological choices adopted in fact strongly determine the success of the proposed solution, as they may imply limitations both to the person who uses it and to the achievable performance. In this document, targeting the case of a fall detection sensor based on a pair of sensorized shoes, the effectiveness of a real implementation of an Internet of Things technology is examined. It is shown how alarming events, generated in a metropolitan context, are effectively sent to a supervision system through Low Power Wide Area Network technology without the need for a portable gateway. The experimental results demonstrate the effectiveness of the chosen technology, which allows the user to take advantage of the support of a wearable sensor without being forced to substantially change his lifestyle.
\end{abstract}

Index Terms-Wireless sensor network, low power, LoRa, fall detection, wearable device.

\section{INTRODUCTION}

In recent years, several research studies have tried to propose technological solutions capable of supporting frail elderly people with the aim of limiting the decay of their degree of independence. Focusing, as an example, on fall detection, that represent a major challenge in the elderly population, Mubashir et al. in [1] classify the systems and algorithms according to three general categories: ambient sensors-based, vision-based, and wearable device-based detection systems. The ambient sensors-based approach often includes presence, vibration, pressure, or acoustic sensors [2], [3]. It represent a kind of systems striving to embed sensors in the living environment to recognize the falls in a totally non-intrusive

Manuscript received March 27, 2020; revised April 25, 2020. Date of publication July 21, 2020. Date of current version July 21, 2020. The associate editor prof. Dinko Begušić has been coordinating the revew of this manuscript and approved it for publication.

D. Disha, S. Spinsante and E. Gambi are with the Dipartimento di Ingegneria dell'Informazione, Università Politecnica delle Marche, 60131 Ancona, Italy (e-mail: d.disha@pm.univpm.it, s.spinsante@univpm.it, e.gambi@univpm.it).

Elma Zanaj (corresponding author) is with the Department of Electronic and Telecommunications, Faculty of Information Technology, Polytechnic University of Tirana, Tirana, Albania (e-mail: ezanaj@fti.edu.al).

Digital Object Identifier (DOI): 10.24138/jcomss.v16i3.1039 way, while keeping the user safe from privacy issues. Visionbased systems, on the contrary, exploit one or more sensors, as fixed RGB cameras, RADAR and depth sensors, to capture images, video frames or reflected signal streams. Through suitable processing algorithms, they can detect shape changes, inactivity, or 3D head motions [1], [4], in order to detect falls.

However, both ambient devices and vision-based systems suffer from two major disadvantages: first, they must be installed in the living environment by technical operators (with a consequent cost increase), and second their use is limited to the environment in which they are located, where they may even suffer limitations due to occlusions. To overcome these limitations, many studies propose the use of wearable devices for fall detection [5]. Typically, the wearable-based approach counts on clothing or accessories to wear, equipped with sensors that can get information about movement, position, or physiological parameters of the wearer. These objects are usually connected to the external world, sending notifications to caregivers or health operators in case of alarm, but also allowing the long-term monitoring via web-accessible platforms. They are part of an emerging market segment, called Wearable Internet of Things (WIoT) [6]. Such systems are rarely standalone as they have limited computing capabilities and communication bandwidth. Therefore, one of the issues that should not be underestimated is the transmission technology used to connect the device to the outside world. In the literature, most solutions propose to use short-range transmission technologies, leveraging the smartphone as a portable gateway towards the cloud or the remote control platform. Nevertheless, studies demonstrate that the percentage of mobile phone ownership in the elderly population is still low. For example, in [7], the authors affirm that only $18 \%$ of the 570 people over 65 years involved in the study have a smartphone.

In this paper, we present an Internet of Things (IoT) sensor for fall detection, able to transmit the alarming event to a supervising system, through the Long-Range technology (LoRa) [8], that allows the wearable device to send the information remotely, without leaning on an intermediate portable gateway. Among the different communication technologies used for Low Power Wide Area Networks (LPWAN), LoRa offers the best compromise solution, both in terms of device costs and communication performance [9]. The proposed solution, which integrates all the electronics necessary for the acquisition of the data generated by the sensing elements, their processing and the transmission of the processed data inside 
a shoe, does not change the user's life habits, which can take advantage of the technological support of the fall detection sensor simply by putting on his/her shoes before leaving home. The aim of the work is therefore to demonstrate the IoT capabilities of the sensor, already developed in a prototype way and tested in the laboratory, in a real scenario. The paper is structured as follows. Section II overviews the available literature on the technologies for wearable-based fall detection systems, paying particular attention to the relationship between the adopted architectures and the communication technologies used. Section III describes the IoT communication protocols and, especially, the LoRa technology and LoRa Wide Area Network (LoRaWAN) protocol. The architecture of the proposed system, along with its hardware and software components, is described in Section IV, while the results obtained in the experimental tests are presented in Section V, and discussed in Section VI. Finally, Section VII draws the main conclusion of the work.

\section{BACKGROUND}

The analysis of the literature relating to wearable sensors shows the correlation existing between the objectives of the monitoring to be implemented and the communication technologies and the sending of alarm messages that are adopted. However, as shown below, the proposed solutions either rely on short-range transmission technologies, the use of which in outdoor environments necessarily requires the presence of a gateway (often a smartphone) or directly use the sensors on the smartphone to detect the monitored activity, which is then notified through the smartphone itself.

In [10], the design and implementation of a wearable fall detection system for people affected by Parkinson's disease (PD) are presented, based on the low-power ZigBee wireless communication technology for sensor networks. The sensing system relies on different sensors, including four tilt switches with low power configuration, an accelerometer, an Electromyography (EMG) sensor to be placed on the subject's leg posterior muscle, a Force Sensing Resistor (FSR) placed in the shoe, under the metatarsal head. Despite the positive performance presented by the authors, the usability and compliance of the proposed device are questionable, considering the specific users' needs and requirements. Additionally, differently from the solution presented here, the system proposed in [10] can be used only in a short-range communication scenario, as it is based on the activation of a buzzer alarm on a receiving device carried by a family caregiver. A wearable system exploiting four surface EMG electrodes and 3 FSRs located on the subject's insole is presented in [11] as well, for the aim of daily activity monitoring and fall detection. The purpose of this work is mainly to design appropriate data processing algorithms to ensure high classification accuracy. As a matter of fact, even if a prototype sensing system is developed to be worn by the subjects under test, it is not enabled by a wireless data communication interface. A more recent review of wearable fall detection systems is given in [12], where the focus is mostly on the machine learning algorithms adopted together with smart wrist-bands, that are assumed to be more compliant to the users' needs, especially the older adults' ones. The review criticizes the use of smartphones with apps as wearable fall detection systems, as it is not possible to ensure that the users have the device in their pockets all day long, and this condition is far less probable than having a user wearing a wristband. The same review mentions an interesting work on fall detection and human activity classification by Yacchirema et al. [13]. A 3D-axis accelerometer embedded into a 6LowPAN wearable device provides data from movements of elderly people in realtime. In order to ensure a high efficieny of the fall detection system, the sensor readings are processed and analyzed using a decision trees-based Big Data model running on a Smart IoT Gateway. If a fall is detected, an emergency alert is raised and delivered to different identified actors, exploiting a WiFi connection from the Gateway to the internet. The presence of the Gateway in charge of performing the high-demanding computing processes on the collected data and the fact that the wearable device communicates with the Gateway on a 6LowPAN link limits the applicability of the proposed solution to indoor scenarios. The system presented in this paper, on the contrary, aims for a solution that can track the user's condition outside, even at a large distance from the receiving terminal.

Kerdjidj et al. [14] exploit a wearable Shimmer device to transmit some inertial signals via a short-range wireless connection to a computer, where decision tree algorithms are applied to detect falls. In order to reduce the size of the transmitted data and minimize the energy consumption, a Compressive Sensing (CS) method is applied. The Shimmer wearable technology is applied in the work by Mehmood et al. [15] too, in which a fall event is identified by resorting to the use of the Mahalanobis distance on real-time data. The proposed algorithm is tested and validated on a dataset collected by the authors and including three daily life activities, such as walking, sitting (on) and getting up (from) a chair, and standing still. They are identified as the Activities of Daily Living (ADLs) most frequently associated to fall events in elderlies. Shimmer is a research-oriented device that, differently from the solution proposed in this work, does not have a long-range data transmission capability and can even create stigma in the user, being far from having the appearance of a consumer electronics device. The target of the system herein described, on the contrary, is to attain a wearable device with long-range data transmission capability, possibly integrated with common objects like the shoes any subject would normally wear.

A real-time fall detection system based on the acceleration sensor available onboard smartphones is presented in [16]. The communication capabilities of the smartphone are exploited to locate the position of the user, through the real-time location tracking function enabled by the Google Map's service, in order to eventually raise an alarm in the case of a fall event. For sure, the diverse sensors and communication interfaces available in smartphones make them ideally suitable to applications related to fall detection, however, when considering real life constraints (such as the fact that elderly people often do not possess or carry a smartphone in their pockets, or they can just forget to have the smartphone with them when walking 
and possibly falling, especially indoor), its effectiveness in coping with fall detection becomes less clear and reliable. As a consequence, a solution based on true wearable technologies may better fit the practical operational conditions.

The use of wearable devices poses then several challenges. On the one hand, they offer the chance to monitor and follow the users in every situation of their daily life, while, on the other, such an approach forces them to wear the device constantly, causing acceptability issues. Studies suggest that most of the elderly people do not want to be seen wearing health monitoring devices [17], not to mention concerns about fashion and aesthetics [18]. For these reasons, in recent years, numerous efforts have been made to realize as unobtrusive as possible wearable systems, embedding them in textile vests, bracelets, necklaces or rings [19]. Such small devices, however, do not allow complex processing procedures, due to limited computational resources. On the other hand, embedding the analysis and classification module into the wearable undoubtedly results in a more robust and responsive system, since wireless communications are often unreliable, and may be unavailable or prone to errors [20].

A further problem affecting the wearables regards the energy consumption, since the improvement of battery technology is not as fast as the developments in the digital processing and radio frequency integration [21]. As well known, communication is usually the most energy demanding operation. For this reason, it is necessary to conceive new strategies to minimize the amount of transmitted data. According to Delahoz and Labrador in [20], there exist different methods to limit the power consumption, such as data aggregation and compression, or by performing data analysis and classification onboard. Anyway, short range technologies (Bluetooth, Wi$\mathrm{Fi}$, etc.) should be preferred over long range ones (Cellular, WiMAX, etc.), since they use less power [20]. Nevertheless, this implies that the receiving node is located closely to the wearable. For this reason, some solutions implemented in literature can be used by the subject in a limited spatial range, while others require a mobile gateway to be carried.

The sensing function is obviously performed by the wearable, while the reasoning one can be run onboard or by another device, such as the integration device or the remote platform. Out of the considered research works, in most part of cases, the device that performs the sensing function coincides with the one that recognizes the falls and, therefore, the communication of raw data, characterized by a quite high throughput, is not required. In such cases, the communication takes place mainly with the notifying device or directly with the caregivers. However, to transmit both raw and processed data, most solutions utilize short range technologies, especially Bluetooth, Bluetooth Low Energy (BLE), and ZigBee.

Lastly, the notification management module can be fed, directly, from the reasoning, or, indirectly, from the storage and monitoring module. As shown in Table I, most papers use the smartphone to send notifications in the form of voice calls or text messages.

\section{IOT COMMUNiCATION TECHNOLOGIES}

Wearable IoT (WIoT) has been defined by Hiremath et al. as "a technological infrastructure that interconnects wearable sensors to enable monitoring human factors including health, wellness, behaviours and other data useful in enhancing individuals' everyday quality of life" [6].

In the IoT, the communication technology can be classified by the transmission range in short or long range.

As mentioned in Section II, most of the solutions presented in the literature exploit short range technologies: Bluetooth, BLE, and ZigBee are the most popular ones. They cover a range of a few dozen meters and, for this reason, are particularly suitable indoor. For outdoor use, a portable gateway is necessary.

Considering longer ranges, two main groups can be distinguished: cellular networks and Low Power Wide Area Network (LPWAN). The former are very widespread and characterized by a great throughput, although costly both economically and energetically. On the contrary, when sending a few information on long distances, the best choice is represented by the LPWAN solutions. In fact, they penalize the data rate, using a narrow band, in favour of a greater tolerance to interferences and signal attenuation, allowing a coverage of a few to tens kilometres, and a very long battery life [8].

According to Raza et al. in [46], five technologies have gained momentum in this field: SigFox [47], LoRa [48], Ingenu RPMA [49], Telensa [50], and Qowisio [51]. In addition to these, it is worth mentioning the Narrow Band IoT (NBIoT) standard, which represents a key technology in the view of a future 5G-IoT infrastructure [52]. Among the LPWAN solutions, in the following we will focus on LoRa, since it offers two main advantages: bi-directionality and a business model which allows to implement public or private networks. Moreover, Petäjäjärvi et al. demonstrated the feasibility of using such a technology for wearable-based applications, both outdoor [53] and indoor [54].

\section{A. LoRa}

LoRa is a wireless technology aimed for IoT and machineto-machine (M2M) communications, characterized by a long range coverage and low power consumptions patented by Semtech. More specifically, it is a physical layer technology that exploits SubGHz ISM band and a proprietary Spread Spectrum modulation [55]. The spreading technique chosen is the Chirp Spread Spectrum (CSS), that uses chirp pulses to encode the information. However, the CSS modulation used in the LoRa networks is even more tightening and advanced, in order to meet the IoT requirements [56]. LoRa supports multiple Spreading Factors (SF), i.e. from 7 to 12, in order to trade-off data rate and coverage range. Moreover, it exploits a Forward Error Correction (FEC) technique to further increase the receiver sensitivity. The low-speed transmission and the chosen modulation lead to a low sensitivity of the receiver (up to $-142 \mathrm{dBm}$ ). Such a feature, along with the output power of $+14 \mathrm{dBm}$, gives very high link budgets (up to $156 \mathrm{~dB}$ ). These characteristics make it possible to have Line-Of-Sight (LOS) 
TABLE I

OVERVIEW of Methods AND TeChNOLOGIES USED IN THE RELATEd STUdies

\begin{tabular}{|c|c|c|c|c|c|c|c|}
\hline Ref. & Year & Sensors & $\begin{array}{c}\text { Communication } \\
\text { Technology }\end{array}$ & $\begin{array}{l}\text { Reasoning } \\
\text { Device }\end{array}$ & $\begin{array}{l}\text { Storing } \\
\text { Center }\end{array}$ & $\begin{array}{c}\text { Notification } \\
\text { Management } \\
\text { Device }\end{array}$ & $\begin{array}{l}\text { Notification } \\
\text { Technology }\end{array}$ \\
\hline [22] & 2010 & Acc. & - & Smartphone & - & Smartphone & Voice/Text message \\
\hline$[23]$ & 2012 & Acc. & - & Smartphone & - & Smartphone & Voice/Text message \\
\hline [24] & 2014 & Acc. & - & Smartphone & - & Smartphone & SMS \\
\hline [25] & 2015 & Acc. & GSM & Wearable & - & Notification & SMS \\
\hline [26] & 2016 & Acc. & Bluetooth/NFC & Smartphone & Smartphone & Smartphone & Voice/Text message \\
\hline [27] & 2016 & Acc./Gyr. & Bluetooth & Smartphone & Smartphone & Smartphone & Voice/Text message \\
\hline [28] & 2011 & Acc. & ZigBee & Wearable & PC & PC & Desktop Interface \\
\hline [29] & 2011 & Acc./Gyr. & Bluetooth & PC & - & $\mathrm{PC}$ & Desktop Interface \\
\hline$[30]$ & 2011 & Acc. & ZigBee & $\mathrm{PC}$ & - & $\mathrm{PC}$ & Desktop Interface \\
\hline [31] & 2012 & Acc. & ZigBee & PC (offline) & $\mathrm{PC}$ & - & - \\
\hline [32] & 2012 & Acc. & - & PC (offline) & SD card & - & - \\
\hline [33] & 2012 & Acc./Gyr./Mag. & Bluetooth & PC (offline) & $\mathrm{PC}$ & - & - \\
\hline [34] & 2013 & Acc./Gyr. & ZigBee & Sink Node & Sink Node & - & - \\
\hline [35] & 2014 & Acc. & Bluetooth LE & Wearable & Remote Server & Smartphone & SMS \\
\hline [36] & 2014 & Acc./Gyr./Mag./Pulse & Bluetooth & Wearable & Remote Server & Remote Server & - \\
\hline [37] & 2014 & Acc./Gyr./Mag. & ZigBee & PC (offline) & $\mathrm{PC}$ & - & - \\
\hline [38] & 2014 & Acc./Pulse & ZigBee & Wearable/PC & - & - & - \\
\hline [39] & 2014 & Acc./ECG/Temp. & Bluetooth & Smartphone & Remote Server & Smartphone & Voice/Text message \\
\hline [40] & 2015 & Acc./Gyr. & Bluetooth & Smartphone & - & Smartphone & Voice/Text message \\
\hline [41] & 2015 & Acc./Gyr./Mag. & Bluetooth & Wearable & PC/Tablet & - & - \\
\hline [42] & 2015 & Acc./Gyr. & Custom & PC (offline) & $\mathrm{PC}$ & - & - \\
\hline [43] & 2016 & Acc. & Bluetooth LE & Smartphone & Cloud & Smartphone & Email/SMS/Calls \\
\hline [44] & 2016 & Acc./Gyr./Mag./Alt. & Bluetooth & PC (offline) & $\mathrm{PC}$ & - & - \\
\hline [45] & 2017 & Acc./Gyr. & Bluetooth & Smartphone & - & Smartphone & Voice/Text message \\
\hline
\end{tabular}

connections within a range of $20 \mathrm{~km}$, or non-LOS (NLOS) connections up to $2 \mathrm{~km}$ in urban environments [57].

\section{B. LoRaWAN}

As discussed hitherto, LoRa describes a physical layer that enables the long-range communication link. Nevertheless, it does not define the higher layers, nor even the network architecture. For this reason, the LoRa Alliance proposed an open standard, named LoRa Wide Area Network (LoRaWAN), defining both the architecture and communication protocol, built upon the LoRa physical layer [8].

The architecture of a typical LoRaWAN network is represented in Figure 1. It mainly features three elements:

- end nodes: they communicate the information to the gateway through the LoRa technology;

- gateway: it acts as a concentrator that transparently forwards the data received from the end nodes to the network server, and vice versa;

- network server: it deals with decoding the arriving packets and generating replies. It is also responsible for choosing the best gateway, frequency, and data rate.

LoRa can be used in private and public networks. In both cases, it requires the presence of a base station (gateway) acting as the center node of a star topology. The number of nodes connected to the base station depends on the application and, more precisely, on the number of packets that must be transmitted in a given period of time. Actually, the LoRaWAN topology can be defined as a "star-of-stars" [46], as each message transmitted by the end device is received by all the base stations in the range. This way, the reception diversity can improve the packet delivery ratio, and provide information on the transmitter location. Communication to end point nodes

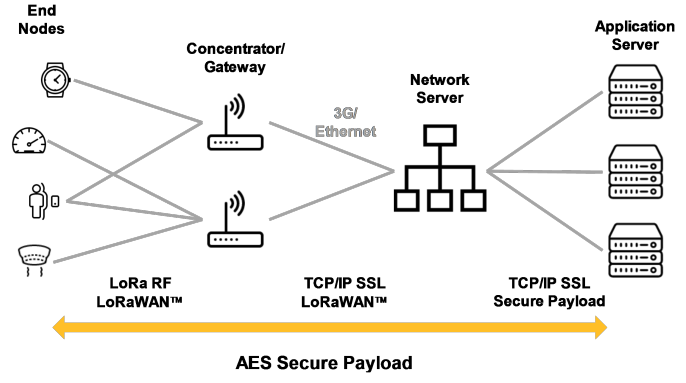

Fig. 1. A general LoRaWAN architecture according to [8].

is generally bi-directional, but it is also possible to support multicast operations.

Basically, LoRaWAN networks exploit the ALOHA method. Such a method is asynchronous and enables the communication when the nodes have something to send, reducing the battery consumption due to synchronization messages. Moreover, LoRaWAN copes with the adaptation of data transmission rate and output power, in order to optimize signal strength and battery consumption. By means of the Adaptive Data Rate (ADR), the network suggests the best SF to use to the end node.

Finally, the LoRaWAN ensures the node authenticity, and the message security through the AES encryption with a key exchange utilizing an IEEE EUI64 identifier [8].

\section{The Proposed Fall Detection System}

As already mentioned in Section I, the system designed by the authors and presented in this paper exploits a pair of instrumented shoes to detect falls and notify such events to 
a supervising system. The system architecture is depicted in Figure 2. The smart shoes are equipped with force sensors and accelerometers in order to analyse the distribution of body weight on the soles, and the feet's inclination angles with respect to the ground. The fall detection is implemented by a board embedded in the bottom of the shoe, which is also able to send the fall alarm to the network server through the LoRa technology. The network server is designed to store the received messages, and deals also with the notification management. In other words, it is able to send the alarming event information to a caregiver, and keeps in memory a history of past messages. The following subsections describe the hardware and software components of the system.

\section{A. Wearable Device}

In the proposed system, the sensing device is represented by an original pair of smart shoes. A shoe prototype is shown in Figure 3(a), while its hardware components are shown in Figure 3(b).

A first version of the prototype device was presented in [58] and [59]. In those cases, however, the purpose of the application was to monitor the vitality level of the elderly, by transmitting information on the gait cycle to the smartphone via BLE. Each shoe is equipped with an instrumented insole. Three Force Sensing Resistors (FSRs) are applied on the insole, placed in correspondence to the heel, 1st, and 5th metatarsal heads. They allow to acquire information on the gait cycle phase, distinguishing among heel contact $(\mathrm{H})$, flat foot $(\mathrm{F})$, push off $(\mathrm{P})$, and limb swing $(\mathrm{S})$. The shoe is also equipped with a triaxial accelerometer embedded in a hole dug in the bottom of the shoe. It enables the foot orientation recognition, through the calculation of pitch and roll angles. The fall detection algorithm works independently for each shoe. It is supplied with data acquired by the accelerometer and the FSRs, and produces as output an alarm message. By combining the information on the foot orientation with the gait cycle phase, it is possible to distinguish an unusual position of the foot, and thus determine the fall. The algorithm is also able to detect recoveries. When a fall or a recovery occurs, the shoe sends the information to the LoRa gateway. A deeper description of algorithm and fall recognition performances is reported in [60]. Fall and recovery messages are characterized by a header of 13 byte, as required by the LoRaWAN protocol,

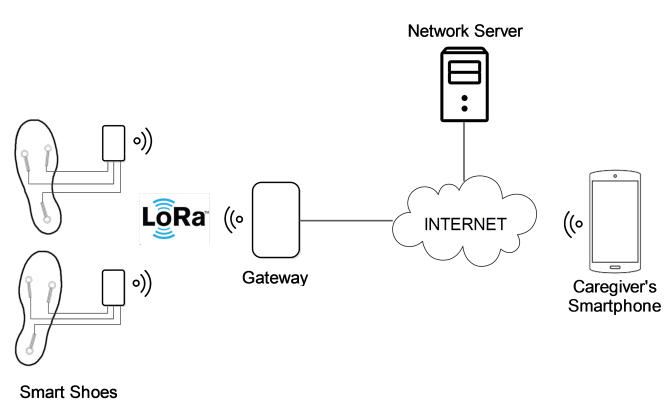

Fig. 2. System architecture.

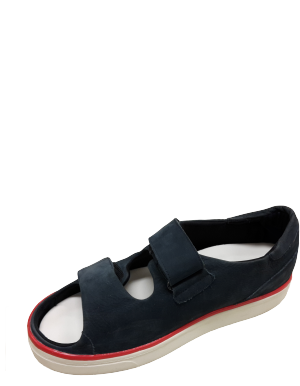

(a) Shoe prototype

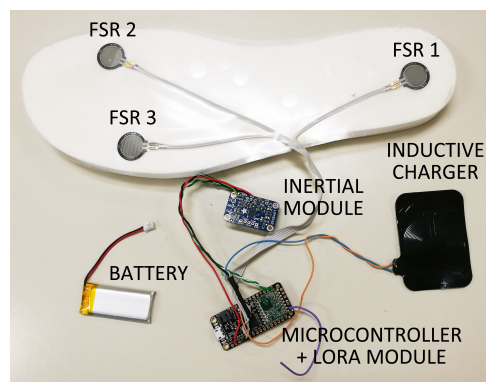

(b) Hardware components
Fig. 3. (a) The shoe prototype and (b) its hardware components.

and by a payload of 14 and 18 byte, respectively. The payload features the following format:

SHOES:<alarm type> <relative direction>.

where $<$ alarm type $>$ denotes the contents of the alarm message and can assume the values "FALL" or "RECOVERY", while <relative direction> identifies the shoe that is sending the message and therefore can be "lx" for the left or "rx" for the right.

As regards communication, in the market there are many evaluation boards that implement the LoRa standards. The board chosen is the Adafruit Feather M0 with RFM95 LoRa Radio [61]. It is small $(51 \mathrm{~mm} \times 23 \mathrm{~mm} \times 8 \mathrm{~mm})$ and lightweight $(5.8 \mathrm{~g})$ enough to be embedded in the footwear in a unobtrusive way. The board is equipped with a microcontroller, which implements the signal acquisition, fall detection, LoRaWAN protocol, and data transmission procedures.

The system is also provided with a rechargeable battery, and an inductive charger, which enables the wireless battery charging. An inductive coil is installed in the stiffener of the shoe, thereby the battery charging can simply be performed by inserting a charging pipe in the shoe.

The battery, the board and the accelerometer are all embedded in the bottom of the shoe and covered by a lid. This way the user can walk comfortably, whitout noticing the presence of the hardware, while facilitating the maintenance.

\section{B. Gateway}

The gateway used in this system, realized by the authors, is composed by a radio module connected through the Serial Peripheral Interface (SPI) to a host, which enables the UDP connection towards the network server. The radio module deals with all the communications based on LoRa technology, while radio message processing, as well as protocol-related tasks, are carried out by an external host.

In order to implement a multi-channel and bidirectional LoRaWAN network, we realized a gateway, built by connecting an iC880A board by IMST GmbH to a Raspberry Pi via SPI. The iC880A board integrates two Semtech SX1257 transceivers and an SX1301 baseband processor: this combination allows to emulate 49 LoRa demodulators with 10 parallel demodulation paths, with the aim to receive up to 8 LoRa packets simultaneously sent with different SFs and on 


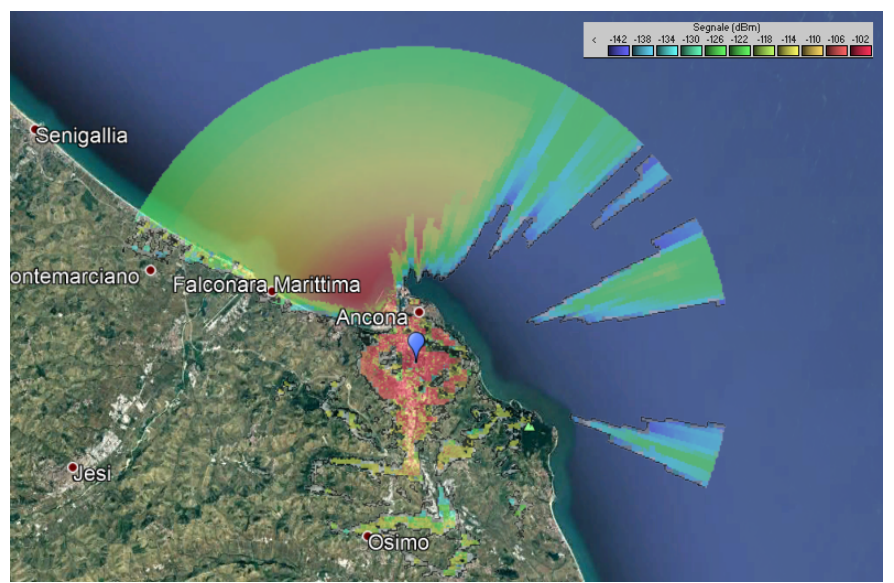

Fig. 4. Estimated coverage area using a single gateway (blue flag) on top of the tower building at at Università Politecnica delle Marche, Ancona (Italy).

different channels. In fact, the SX1257 transceiver integrates an I/Q modulator/demodulator supporting different modulation schemes, including LoRa technology, while the SX1301 digital baseband chip is a powerful digital signal processing engine, specifically designed to provide gateway functionality in the ISM band.

Regarding the software component, the Raspberry Pi runs a modified version of the packet_forwarder program, developed by Semtech. It allows to forward the messages received from the gateway to our network server (described below), and vice versa, exploiting the UDP protocol.

\section{Network Server}

Since the LoRaWAN network server is proprietary, we realized a private network server. It deals with the GWMP (Gateway Message Protocol) communication towards the gateway, but also copes with the packets managing and processing, and with the creation of acknowledgement packets in downlink. In fact, when a fall event occurs, the shoes transmit the message repeatedly until they receive a confirmation reply. Received packets containing relevant information (i.e. fall and recovery), are stored in a database and displayed in a web dashboard.

The server is also in charge of the notification management. To this aim, we foresee to integrate a MQTT middleware. This way, the server can promptly forward the alarm messages to a caregiver, as already done in [62].

\section{EXPERIMENTAL RESULTS}

In order to evaluate the effectiveness of the proposed method we conducted several experiments in the outdoor environment. Tests involved the creation of a single-star private network where data is exchanged with the gateway via LoRa. The gateway, in its turn, communicates with a network server using a User Datagram Protocol (UDP) and an intermediate network (Ethernet or WiFi). The server deals with data management and processing, and, through the gateway, responds to the shoes, which require a receipt confirmation.

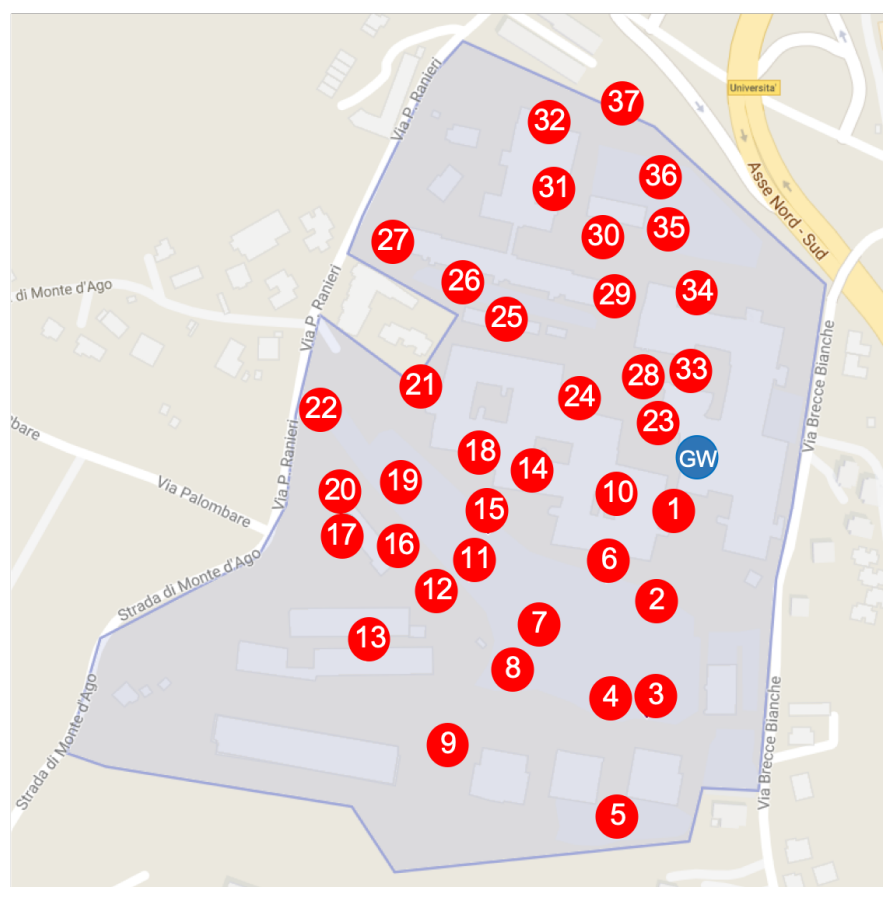

Fig. 5. PoMs and GW location: the blue flag indicates the gateway, while red flags 1 to 37 represent the different positions of the end nodes. The University Campus area is highlighted in grey.

\section{A. Preliminary Radio Coverage Evaluation}

In order to estimate the radio coverage range in the urban environment, we conducted a preliminary study. Through Radio Mobile software, we simulated the radio coverage of the LoRa technology in the city of Ancona (Italy), using the following parameters:

- TX power: $14 \mathrm{dBm}$;

- frequency: from 867.1 MHz to $868.5 \mathrm{MHz}$ (8 channels spaced by $200 \mathrm{kHz}$ );

- RX sensitivity: $-142 \mathrm{dBm}$;

- RX antenna gain: $0 \mathrm{dBi}$;

- RX antenna polarization: vertical;

- RX antenna polar pattern: omnidirectional;

- RX antenna height above sea level: 200 m;

- statistical margin value added to link's path loss: $14.8 \mathrm{~dB}$.

The gateway is located on the top of the tower building of the Engineering Faculty, at Università Politecnica delle Marche, in Ancona. The tower is about $40 \mathrm{~m}$ high and is built on a hill, at $160 \mathrm{~m}$ above the sea level.

The obtained radio coverage map is shown in Figure 4. Red areas are the ones which provide the higher Received Signal Strength Indicator (RSSI) values, while blue areas are the ones featuring the worst coverage.

\section{B. Tests in the University Campus}

We conducted a first experimental evaluation of the actual outdoor radio coverage, by sending simulated fall messages from different locations. The test campaign was held in the University Campus, by placing the LoRa gateway on the top of the tower building of the Engineering Faculty. 
In Figure 5, we present a map of the Points of Measure (PoM). Table II lists, for each of them, the estimated distance between the shoes' location and the gateway's antenna, as well as the respective number of transmitted and received packets and the calculated packet success ratio. The distribution of RSSI values is represented in Figure 6.

The University Campus is built on a hill, and, therefore, distributed over several altitude levels. Moreover, the area is mainly covered by buildings and trees. For this reason the RSSI values have a non-linear trend over the distance from the gateway. However, as can be seen from Table II, the presented results indicate that $95 \%$ of all the data packets were successfully transferred for the selected locations.

\section{Tests in the Urban Environment}

A second experimental evaluation was conducted in the urban environment. Also in this case, the LoRaWAN network was created by placing the gateway on the top of the tower building. During the tests, we simulated falls in 6 different locations of the city (see Figure 7). From each PoM, we transmitted 5 fall messages using a SF of 7 , and 5 fall messages using a SF of 12. The average RSSI values received by the gateway for packets having the same SF is reported in Table III. For each PoM, the table shows also the GPS coordinates, a brief description of the location, and the distance from the gateway.

The average RSSI values received from the first two PoMs are optimal, especially in the first case, since during the measurement the end node was in LOS. In the 3rd and 5th cases, the tests took places in urban streets surrounded by buildings. As a result, the receiver lost some packets with a SF of 7. Despite the greater distance, in case 4, all the packets reached the gateway with a fair RSSI. In fact, in such a case the end node was placed on the top of a hill, in LOS with the base station. The last test provided the worst results: no message reached the destination. This is certainly due to unfavourable position of the transmitting device. In fact, the radio link was
TABLE II

RESULTS OF THE FIRST TEST CAMPAIGN IN TERMS OF SUCCESS RATIO.

\begin{tabular}{cccccc}
\hline PoM & Coordinates & Distance $(\mathrm{m})$ & No. of TX packets & No. of RX packets & Success Ratio \\
\hline 1 & 43.58652513 .516407 & 64 & 417 & 410 & $98 \%$ \\
\hline 2 & 43.58600213 .516330 & 115 & 357 & 347 & $97 \%$ \\
\hline 3 & 43.58541913 .516218 & 177 & 424 & 410 & $97 \%$ \\
\hline 4 & 43.58542313 .515975 & 184 & 408 & 383 & $94 \%$ \\
\hline 5 & 43.58475913 .516003 & 252 & 397 & 367 & $92 \%$ \\
\hline 6 & 43.58620513 .515892 & 109 & 416 & 376 & $90 \%$ \\
\hline 7 & 43.58587613 .515337 & 163 & 291 & 283 & $97 \%$ \\
\hline 8 & 43.58560013 .515130 & 210 & 395 & 355 & $90 \%$ \\
\hline 9 & 43.58517013 .514640 & 248 & 282 & 269 & $95 \%$ \\
\hline 10 & 43.58660513 .515959 & 76 & 392 & 378 & $96 \%$ \\
\hline 11 & 43.58621213 .514790 & 174 & 420 & 406 & $97 \%$ \\
\hline 12 & 43.58604813 .514552 & 202 & 387 & 349 & $90 \%$ \\
\hline 13 & 43.58575413 .513961 & 256 & 428 & 408 & $95 \%$ \\
\hline 14 & 43.58675013 .515293 & 114 & 401 & 373 & $93 \%$ \\
\hline 15 & 43.58649113 .514944 & 149 & 393 & 368 & $94 \%$ \\
\hline 16 & 43.58631513 .514209 & 209 & 429 & 412 & $96 \%$ \\
\hline 17 & 43.58641113 .513798 & 240 & 407 & 394 & $97 \%$ \\
\hline 18 & 43.58684913 .514909 & 142 & 417 & 382 & $92 \%$ \\
\hline 19 & 43.58670013 .514251 & 199 & 415 & 394 & $95 \%$ \\
\hline 20 & 43.58660113 .513759 & 237 & 420 & 397 & $95 \%$ \\
\hline 21 & 43.58726913 .514403 & 185 & 416 & 391 & $94 \%$ \\
\hline 22 & 43.58713213 .513600 & 246 & 419 & 394 & $94 \%$ \\
\hline 23 & 43.58704413 .516308 & 51 & 415 & 403 & $97 \%$ \\
\hline 24 & 43.58718513 .515691 & 89 & 415 & 407 & $98 \%$ \\
\hline 25 & 43.58763513 .515096 & 149 & 360 & 352 & $98 \%$ \\
\hline 26 & 43.58785613 .514734 & 186 & 414 & 403 & $97 \%$ \\
\hline 27 & 43.58809713 .514154 & 239 & 418 & 407 & $97 \%$ \\
\hline 28 & 43.58728813 .516234 & 70 & 404 & 395 & $98 \%$ \\
\hline 29 & 43.58776513 .515960 & 117 & 417 & 406 & $97 \%$ \\
\hline 30 & 43.58810813 .515860 & 152 & 384 & 367 & $96 \%$ \\
\hline 31 & 43.58839413 .515478 & 193 & 418 & 403 & $96 \%$ \\
\hline 32 & 43.58839413 .515478 & 234 & 400 & 387 & $97 \%$ \\
\hline 33 & 43.58734113 .516503 & 67 & 274 & 267 & $97 \%$ \\
\hline 34 & 43.58778413 .516632 & 109 & 417 & 396 & $95 \%$ \\
\hline 35 & 43.58817313 .516375 & 151 & 412 & 381 & $92 \%$ \\
\hline 36 & 43.58846713 .516310 & 184 & 412 & 394 & $96 \%$ \\
\hline 37 & 43.58890213 .515993 & 232 & 421 & 411 & $98 \%$ \\
\hline Total & & $\mathbf{6 1 7 5}$ & $\mathbf{1 4 7 1 2}$ & $\mathbf{1 4 0 2 5}$ & $\mathbf{9 5} \%$ \\
\hline & & & & &
\end{tabular}

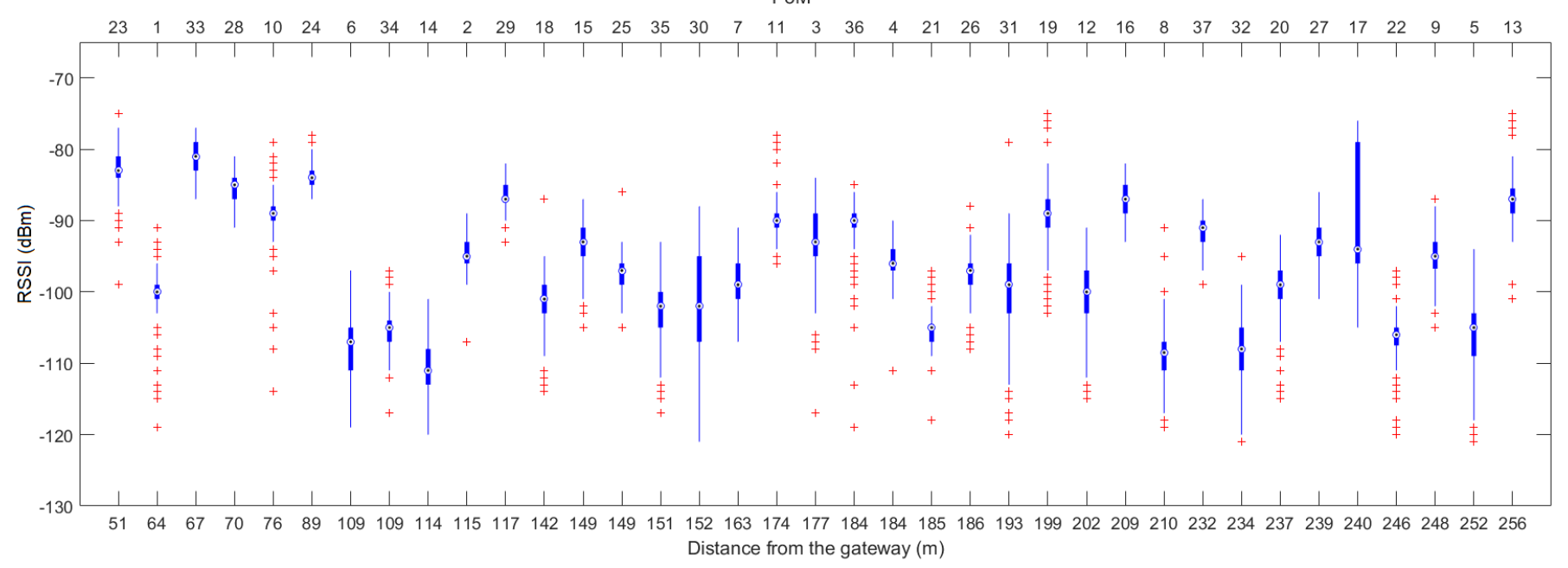

Fig. 6. Box plot of RSSI values for each PoM sorted by the distance from the gateway. 
TABLE III

Results of the Experimental Tests Performed in 6 Different PoMs.

\begin{tabular}{|c|c|c|c|c|c|c|}
\hline PoM & $\begin{array}{c}\text { Coordi- } \\
\text { nates }\end{array}$ & $\begin{array}{c}\text { Distance } \\
(\mathrm{m})\end{array}$ & Description & SF & $\begin{array}{c}\text { RSSI } \\
(\mathrm{dBm})\end{array}$ & $\begin{array}{l}\text { Packets } \\
\text { received }\end{array}$ \\
\hline \multirow{2}{*}{1} & 43.590043 & \multirow{2}{*}{339} & \multirow{2}{*}{ Outdoor parking. LOS link. } & 7 & -95.50 & $5 / 5$ \\
\hline & 13.516866 & & & 12 & -101.67 & $5 / 5$ \\
\hline \multirow[b]{2}{*}{2} & & \multirow[b]{2}{*}{774} & \multirow{2}{*}{$\begin{array}{l}\text { Outdoor parking. } \\
\text { The link is not } \\
\text { completely LOS } \\
\text { due to trees. }\end{array}$} & 7 & -106.25 & $5 / 5$ \\
\hline & 13.522077 & & & 12 & -108.33 & $5 / 5$ \\
\hline \multirow{2}{*}{3} & 43.608285 & \multirow{2}{*}{2390} & \multirow{2}{*}{$\begin{array}{l}\text { Urban street surrounded } \\
\text { by buildings. NLOS link. }\end{array}$} & 7 & -116.72 & $4 / 5$ \\
\hline & 13.513118 & & & 12 & -117.50 & $5 / 5$ \\
\hline \multirow{2}{*}{4} & 43.625085 & \multirow{2}{*}{4280} & \multirow{2}{*}{$\begin{array}{l}\text { Outdoor parking. The area } \\
\text { is on top of a hill. LOS link. }\end{array}$} & 7 & -113.50 & $5 / 5$ \\
\hline & 13.510417 & & & 12 & -117.00 & $5 / 5$ \\
\hline \multirow{2}{*}{5} & & \multirow{2}{*}{3380} & \multirow{2}{*}{$\begin{array}{l}\text { Urban street flanked on one } \\
\text { side by buildings, and on the } \\
\text { other one by the seaport. } \\
\text { NLOS link. }\end{array}$} & 7 & -118.16 & $2 / 5$ \\
\hline & 13.506626 & & & 12 & -119.50 & $5 / 5$ \\
\hline \multirow[b]{2}{*}{6} & 43615740 & \multirow{2}{*}{3200} & \multirow{2}{*}{$\begin{array}{l}\text { Urban street } \\
\text { surrounded by buildings. } \\
\text { The area is down a hill. } \\
\text { NLOS link. }\end{array}$} & 7 & - & $0 / 5$ \\
\hline & 13.513068 & & & 12 & - & $0 / 5$ \\
\hline
\end{tabular}

completely covered by a hill, and the PoM was surrounded by buildings.

Results obtained confirm the simulator's prediction (see Figure 8). In fact, according to simulation results, PoMs 1 and 2 feature a perfect coverage, while 4 and 5 are borderline, and 6 is out of the coverage range. Even though the point 3 is marked as red, it showed results below expectations. The reason is that the simulator takes into account only the soil morphology, and does not consider buildings and trees.

\section{Power Consumption Test}

In order to verify the shoes' power consumption, we performed measures in the laboratory environment. The energy consumption peaks are reported in Table IV for each operation mode. The table shows also the duration. In fact, while the fall detection algorithm is always active, the LoRa transmission occurs only when a fall or recovery event takes place. As expected, depending on the SF, the time duration as well as the energy consumption value are different. LoRa transmission times refer to packets transmitted on a band of $125 \mathrm{kHz}$, a code rate of 4/5, a fixed header of 13 bytes, and a payload of 14 or 18 bytes, respectively for a fall or recovery message. It is interesting to note that the consumption of the fall detection algorithm is just $22 \mathrm{~mA}$. This means that, when using, for example, a $500 \mathrm{~mA} / \mathrm{h}$ battery, the system can run for about 23 hours, i.e. almost a full day. While, when using a $800 \mathrm{~mA} / \mathrm{h}$ battery, the range reaches 36 hours.

\section{DISCUSSION}

In general, the results obtained show that LoRa is an attractive and promising technology in the field of an individual's health and well-being monitoring, as also stated in [53]. In fact, LPWAN technologies allow long-range communication with a low battery consumption burden. However, they are especially suited to application areas in which the amount of transmitted information is limited, such as the fall detection.
TABLE IV

Results of MEASURES ON THE ENERGy CONSUMPTION.

\begin{tabular}{lcc}
\hline Operation mode & $\begin{array}{c}\text { Energy } \\
\text { consumption } \\
\text { peak }\end{array}$ & Time \\
\hline Fall detection algorithm & $22 \mathrm{~mA}$ & Full time \\
\hline LoRa transmission with $\mathrm{SF}=7$ & $118 \mathrm{~mA}$ & $\begin{array}{c}67 \mathrm{~ms} \text { (fall) or } \\
72 \mathrm{~ms} \\
\text { (recovery) }\end{array}$ \\
\hline Lora transmission with $\mathrm{SF}=12$ & $144 \mathrm{~mA}$ & $\begin{array}{c}1483 \mathrm{~ms} \text { (fall) } \\
\text { or 1647 ms } \\
\text { (recovery) }\end{array}$ \\
\hline $\begin{array}{l}\text { Total (fall detection algorithm }+ \\
\text { LoRa transmission) with } \mathrm{SF}=7\end{array}$ & $140 \mathrm{~mA}$ & - \\
\hline $\begin{array}{l}\text { Total (fall detection algorithm }+ \\
\text { LoRa transmission) with } \mathrm{SF}=12\end{array}$ & $166 \mathrm{~mA}$ & - \\
\hline
\end{tabular}

The radio coverage evaluation obtained through the simulator has shown that only one gateway allows to fairly cover most of the city area, providing the fall detection system with the necessary reliability in the alarm messages delivery. Such a claim has been confirmed by the experimental tests carried out, first, in a limited spatial range (the University Campus) and then in the urban environment.

The coverage problems reported during the experiments can be easily solved by installing further gateways in different places. In addition, some studies demonstrate that the presence of multiple (three or more) radio base stations allows the multilateration of the transmitting device and, hence, its localization. For example, Fargas and Petersen in [63], demonstrate that the transmitter's position can be estimated by calculating the Time Difference Of Arrival (TDOA) of a received packet from different gateways. In such a case, the accuracy achieved is about $100 \mathrm{~m}$. This feature is very important in our application area, since it enables the subject's localization, without additional battery consumption (such as that needed to transmit the position data via GPS). This way, 


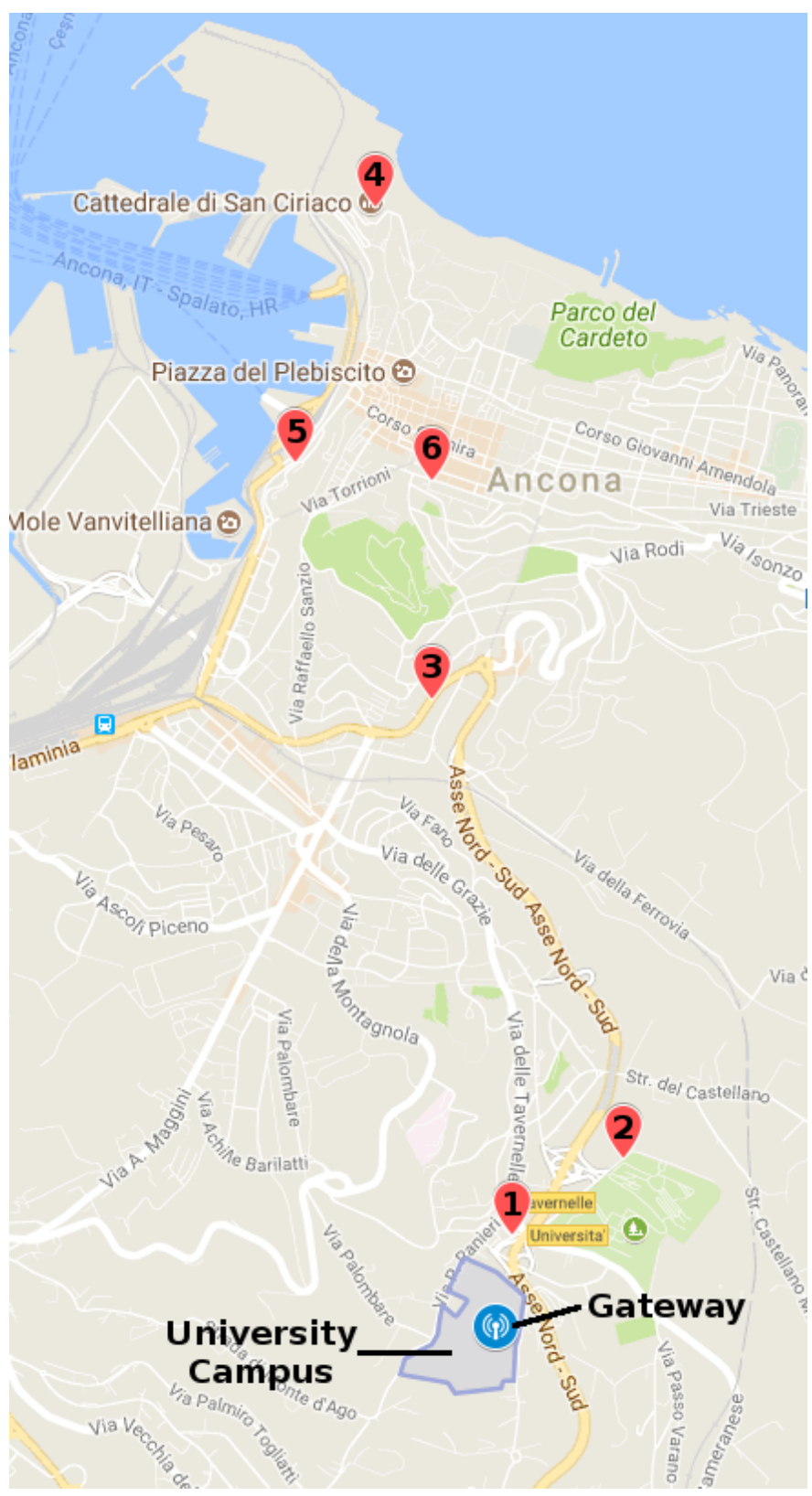

Fig. 7. PoMs and GW location: the blue flag indicates the gateway, while flags 1 to 6 represent the different positions of the end nodes.

the network server can calculate the shoe's position and notify it to the caregiver, along with the fall message.

Experimental results on battery consumption demonstrate that the method and technology chosen are appropriate for this application. In fact, they allow to monitor the elderly and notify alarming events, with low energy consumption. This is of great importance as it guarantees tracking contuinity. In fact, as stated by [64] the battery limit of wearables inhibits users from a continuous tracking of their health status. The system is already geared towards the integration of a notification management module. In this regard, in a previous work [62] we discussed a MQTT-based alert notification system. While in [65], we introduced a system able to acquire LoRa packets and convert them into MQTT messages for building automation purposes.

\section{CONCLUSION}

In this work, the implementation of a wearable sensor for detecting falls is shown experimentally. The authors have verified in a real scenario that the IoT technology adopted allows the sensor to connect to the realized Gateway even over relatively long distances, compatible with metropolitan coverage. Once a fall is detected, the sensor inside the shoe sends a message to the supervisor entity, using a LoRaWAN network. Experimental tests in urban environments demonstrate the effectiveness of the proposed approach. The results show that the chosen transmission technology is adequate to provide the signal strength necessary for the external environment considered. Further improvements include the integration of multiple gateways to improve coverage and the implementation of a functionality to forward information to a potential caregiver.

\section{REFERENCES}

[1] M. Mubashir, L. Shao, and L. Seed, "A survey on fall detection: Principles and approaches," Neurocomputing, vol. 100, pp. 144-152, 2013.

[2] X. Zhuang, J. Huang, G. Potamianos, and M. Hasegawa-Johnson, "Acoustic fall detection using gaussian mixture models and gmm supervectors," in Acoustics, Speech and Signal Processing, 2009. ICASSP 2009. IEEE International Conference on. IEEE, 2009, pp. 69-72.

[3] G. Feng, J. Mai, Z. Ban, X. Guo, and G. Wang, "Floor pressure imaging for fall detection with fiber-optic sensors," IEEE Pervasive Computing, vol. 15 , no. 2, pp. 40-47, 2016.

[4] S. Gasparrini, E. Cippitelli, S. Spinsante, and E. Gambi, "A depth-based fall detection system using a kinect ${ }^{\circledR}$ sensor," Sensors, vol. 14, no. 2 pp. 2756-2775, 2014.

[5] L. Schwickert, C. Becker, U. Lindemann, C. Maréchal, A. Bourke, L. Chiari, J. Helbostad, W. Zijlstra, K. Aminian, C. Todd, et al., "Fall detection with body-worn sensors," Zeitschrift für Gerontologie und Geriatrie, vol. 46, no. 8, pp. 706-719, 2013.

[6] S. Hiremath, G. Yang, and K. Mankodiya, "Wearable internet of things: Concept, architectural components and promises for person-centered healthcare," in Wireless Mobile Communication and Healthcare (Mobihealth), 2014 EAI 4th International Conference on. IEEE, 2014, pp. 304-307.

[7] A. Smith, "Smartphone ownership-2013 update," Pew Research Center: Washington DC, vol. 12, p. 2013, 2013.

[8] L. Alliance, "A technical overview of lora and lorawan," White paper, Nov, 2015.

[9] K. Mekki, E. Bajic, F. Chaxel, and F. Meyer, "A comparative study of lpwan technologies for large-scale iot deployment," ICT Express, vol. 5, no. 1 , pp. $1-7,2019$.

[10] H. A. Hashim, S. L. Mohammed, and S. K. Gharghan, "Accurate fall detection for patients with parkinson's disease based on a data event algorithm and wireless sensor nodes," Measurement, vol. 156, p. 107573, 2020.

[11] X. Xi, W. Jiang, Z. Lü, S. M. Miran, and Z. Luo, "Daily activity monitoring and fall detection based on surface electromyography and plantar pressure," Complexity, vol. 2020, pp. 1-12, 2020.

[12] A. Ramachandran and A. Karuppiah, "A survey on recent advances in wearable fall detection systems," BioMed Research International, vol. 2020, 2020.

[13] D. Yacchirema, J. S. de Puga, C. Palau, and M. Esteve, "Fall detection system for elderly people using iot and big data," Procedia Computer Science, vol. 130, pp. 603 - 610, 2018, the 9th International Conference on Ambient Systems, Networks and Technologies (ANT 2018) / The 8th International Conference on Sustainable Energy Information Technology (SEIT-2018) / Affiliated Workshops.

[14] O. Kerdjidj, N. Ramzan, K. Ghanem, A. Amira, and F. Chouireb, "Fall detection and human activity classification using wearable sensors and compressed sensing," Journal of Ambient Intelligence and Humanized Computing, pp. 1-13, 2019. 


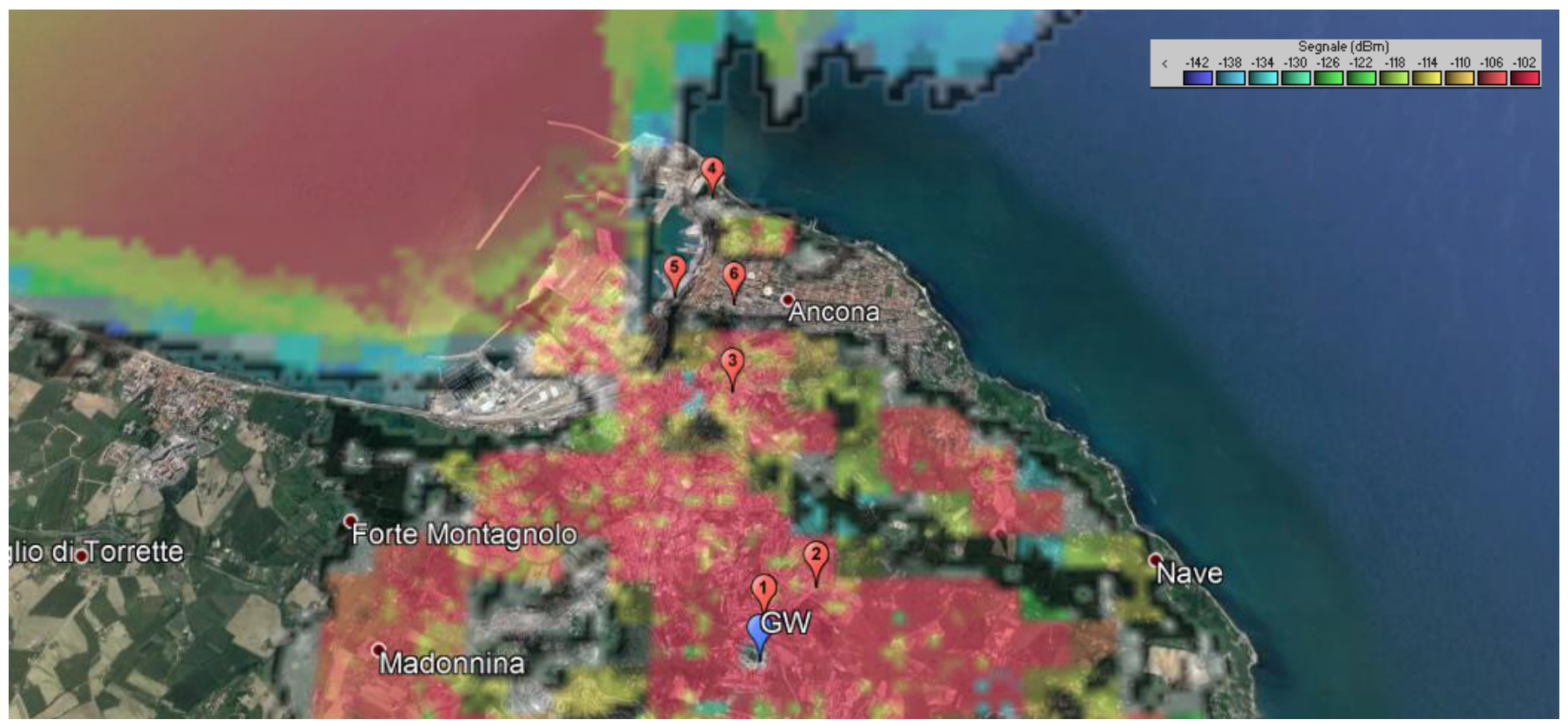

Fig. 8. Estimated coverage area and PoMs.

[15] A. Mehmood, A. Nadeem, M. Ashraf, T. Alghamdi, and M. Siddiqui, "A novel fall detection algorithm for elderly using shimmer wearable sensors," Health and Technology, vol. 9, no. 4, pp. 631-646, 2019.

[16] Y. Lee, H. Yeh, K.-H. Kim, and O. Choi, "A real-time fall detection system based on the acceleration sensor of smartphone," International Journal of Engineering Business Management, vol. 10, p. $1847979017750669,2018$.

[17] R. Steele, A. Lo, C. Secombe, and Y. K. Wong, "Elderly persons' perception and acceptance of using wireless sensor networks to assist healthcare," International journal of medical informatics, vol. 78, no. 12, pp. 788-801, 2009.

[18] V. G. Motti and K. Caine, "Human factors considerations in the design of wearable devices," in Proceedings of the Human Factors and Ergonomics Society Annual Meeting, vol. 58, no. 1. SAGE Publication Sage CA: Los Angeles, CA, 2014, pp. 1820-1824

[19] S. Seneviratne, Y. Hu, T. Nguyen, G. Lan, S. Khalifa, K. Thilakarathna, M. Hassan, and A. Seneviratne, "A survey of wearable devices and challenges," IEEE Communications Surveys \& Tutorials, 2017.

[20] Y. S. Delahoz and M. A. Labrador, "Survey on fall detection and fall prevention using wearable and external sensors," Sensors, vol. 14, no. 10, pp. 19806-19842, 2014

[21] R. Sanchez-Mejias, L. Noël, and H. Holma "Smartphone optimization," LTE Small Cell Optimization: 3GPP Evolution to Release 13, pp. 379421, 2016.

[22] J. Dai, X. Bai, Z. Yang, Z. Shen, and D. Xuan, "Perfalld: A pervasive fall detection system using mobile phones," in Pervasive Computing and Communications Workshops (PERCOM Workshops), 2010 8th IEEE International Conference on. IEEE, 2010, pp. 292-297.

[23] Y. Shi, Y. Shi, and X. Wang, "Fall detection on mobile phones using features from a five-phase model," in Ubiquitous Intelligence \& Computing and 9th International Conference on Autonomic \& Trusted Computing (UIC/ATC), 2012 9th International Conference on. IEEE, 2012, pp. 951-956.

[24] B. Aguiar, T. Rocha, J. Silva, and I. Sousa, "Accelerometer-based fall detection for smartphones," in Medical Measurements and Applications (MeMeA), 2014 IEEE International Symposium on. IEEE, 2014, pp. $1-6$.

[25] F. Wu, H. Zhao, Y. Zhao, and H. Zhong, "Development of a wearablesensor-based fall detection system," International journal of telemedicine and applications, vol. 2015, p. 2, 2015.

[26] L. Basili, G. DeMaso-Gentile, C. Scavongelli, S. Orcioni, S. Pirani, and M. Conti, "Fall detection using wearable accelerometers and smartphone," in Mobile Networks for Biometric Data Analysis. Springer 2016, pp. 299-311.

[27] J. He, M. Zhou, X. Wang, and Y. Han, "A wearable method for autonomous fall detection based on kalman filter and k-nn algorithm," in Biomedical Circuits and Systems Conference (BioCAS), 2016 IEEE. IEEE, 2016, pp. 420-423.

[28] D. Chen, W. Feng, Y. Zhang, X. Li, and T. Wang, "A wearable wireless fall detection system with accelerators," in Robotics and Biomimetics (ROBIO), 2011 IEEE International Conference on. IEEE, 2011, pp. 2259-2263.

[29] O. Ojetola, E. I. Gaura, and J. Brusey, "Fall detection with wearable sensors-safe (smart fall detection)," in Intelligent Environments (IE). 2011 7th International Conference on. IEEE, 2011, pp. 318-321.

[30] M. Yuwono, S. W. Su, and B. Moulton, "Fall detection using a gaussian distribution of clustered knowledge, augmented radial basis neuralnetwork, and multilayer perceptron," in Broadband and Biomedical Communications (IB2Com), 2011 6th International Conference on. IEEE, 2011, pp. 145-150.

[31] S. Z. Erdogan and T. T. Bilgin, "A data mining approach for fall detection by using k-nearest neighbour algorithm on wireless sensor network data," IET Communications, vol. 6, no. 18, pp. 3281-3287, 2012.

[32] A. Sengto and T. Leauhatong, "Human falling detection algorithm using back propagation neural network," in Biomedical Engineering International Conference (BMEiCON), 2012. IEEE, 2012, pp. 1-5.

[33] A. Sorvala, E. Alasaarela, H. Sorvoja, and R. Myllylä, "A two-threshold fall detection algorithm for reducing false alarms," in Medical Information and Communication Technology (ISMICT), 2012 6th International Symposium on. IEEE, 2012, pp. 1-4.

[34] W.-S. Baek, D.-M. Kim, F. Bashir, and J.-Y. Pyun, "Real life applicable fall detection system based on wireless body area network," in Consumer Communications and Networking Conference (CCNC), 2013 IEEE. IEEE, 2013, pp. 62-67.

[35] R. Freitas, M. Terroso, M. Marques, J. Gabriel, A. T. Marques, and R. Simoes, "Wearable sensor networks supported by mobile devices for fall detection," in SENSORS, 2014 IEEE. IEEE, 2014, pp. 2246-2249.

[36] Z. Li, A. Huang, W. Xu, W. Hu, and L. Xie, "Fall perception for elderly care: A fall detection algorithm in smart wristlet mhealth system," in Communications (ICC), 2014 IEEE International Conference on. IEEE, 2014, pp. 4270-4274.

[37] A. T. Özdemir and B. Barshan, "Detecting falls with wearable sensors using machine learning techniques," Sensors, vol. 14, no. 6, pp. 10691 10708,2014

[38] J. Wang, Z. Zhang, B. Li, S. Lee, and R. Sherratt, "An enhanced fall detection system for elderly person monitoring using consumer home networks," IEEE transactions on consumer electronics, vol. 60 , no. 1 , pp. 23-29, 2014

[39] W.-J. Yi, O. Sarkar, S. Mathavan, and J. Saniie, "Wearable sensor data fusion for remote health assessment and fall detection," in Elec 
tro/Information Technology (EIT), 2014 IEEE International Conference on. IEEE, 2014, pp. 303-307.

[40] E. Casilari and M. A. Oviedo-Jiménez, "Automatic fall detection system based on the combined use of a smartphone and a smartwatch," PloS one, vol. 10, no. 11, p. e0140929, 2015.

[41] P. Pierleoni, A. Belli, L. Palma, M. Pellegrini, L. Pernini, and S. Valenti, "A high reliability wearable device for elderly fall detection," IEEE Sensors Journal, vol. 15, no. 8, pp. 4544-4553, 2015.

[42] N. Shibuya, B. T. Nukala, A. Rodriguez, J. Tsay, T. Nguyen, S. Zupancic, and D. Y. Lie, "A real-time fall detection system using a wearable gait analysis sensor and a support vector machine (svm) classifier," in Mobile Computing and Ubiquitous Networking (ICMU), 2015 Eighth International Conference on. IEEE, 2015, pp. 66-67.

[43] I. Maglogiannis, C. Ioannou, and P. Tsanakas, "Fall detection and activity identification using wearable and hand-held devices," Integrated Computer-Aided Engineering, vol. 23, no. 2, pp. 161-172, 2016.

[44] A. M. Sabatini, G. Ligorio, A. Mannini, V. Genovese, and L. Pinna, "Prior-to-and post-impact fall detection using inertial and barometric altimeter measurements," IEEE transactions on neural systems and rehabilitation engineering, vol. 24, no. 7, pp. 774-783, 2016.

[45] J. Santiago, E. Cotto, L. G. Jaimes, and I. Vergara-Laurens, "Fall detection system for the elderly," in Computing and Communication Workshop and Conference (CCWC), 2017 IEEE 7th Annual. IEEE, 2017, pp. 1-4.

[46] U. Raza, P. Kulkarni, and M. Sooriyabandara, "Low power wide area networks: An overview," IEEE Communications Surveys \& Tutorials, vol. 19, no. 2, pp. 855-873, 2017.

[47] Sigfox - the global communications service provider for the internet of things (iot). [Online]. Available: http://www.sigfox.com/en

[48] L. Alliance. Lora technology. [Online]. Available: https://www.loraalliance.org/What-Is-LoRa/Technology

[49] Ingenu - dedicated machine connectivity for iot. [Online]. Available: https://www.ingenu.com/

[50] Telensa. Telensa - smart city iot powered by connected street lighting. [Online]. Available: http://www.telensa.com/

[51] Qowisio. Qowisio - internet of things : Créons ensemble une expérience inédite. [Online]. Available: https://www.qowisio.com/en/

[52] L. Militano, A. Orsino, G. Araniti, and A. Iera, "Nb-iot for d2d-enhanced content uploading with social trustworthiness in $5 \mathrm{~g}$ systems," Future Internet, vol. 9, no. 3, p. 31, 2017

[53] J. Petäjäjärvi, K. Mikhaylov, M. Hämäläinen, and J. Iinatti, "Evaluation of lora lpwan technology for remote health and wellbeing monitoring," in Medical Information and Communication Technology (ISMICT), 2016 10th International Symposium on. IEEE, 2016, pp. 1-5.

[54] J. Petäjäjärvi, K. Mikhaylov, R. Yasmin, M. Hämäläinen, and J. Iinatti, "Evaluation of lora lpwan technology for indoor remote health and wellbeing monitoring," International Journal of Wireless Information Networks, pp. 1-13, 2017.

[55] F. Sforza, "Communications system," Mar. 26 2013, uS Patent $8,406,275$.

[56] Semtech. An1200.22 lora modulation basics. [Online]. Available: http://www.semtech.com/images/datasheet/an1200.22.pdf

[57] A. R. Biswas, C. Dupont, and C. Pham, "Iot, cloud and bigdata integration for iot analytics," Building Blocks for IoT Analytics, p. 11, 2016.

[58] A. D. Santis, A. D. Campo, E. Gambi, L. Montanini, G. Pelliccioni, D. Perla, and S. Spinsante, "Unobtrusive monitoring of physical activity in aal - a simple wearable device designed for older adults," in Proceedings of the 1st International Conference on Information and Communication Technologies for Ageing Well and e-Health, 2015, pp. 200-205.

[59] A. Del Campo, L. Montanini, D. Perla, E. Gambi, and S. Spinsante, "Ble analysis and experimental evaluation in a walking monitoring device for elderly," in Personal, Indoor, and Mobile Radio Communications (PIMRC), 2016 IEEE 27th Annual International Symposium on. IEEE, 2016, pp. 1-6.

[60] L. Montanini, A. Del Campo, D. Perla, S. Spinsante, and E. Gambi, "A footwear-based methodology for fall detection," IEEE Sensors Journal, vol. 18, no. 3, pp. 1233-1242, 2018.

[61] Adafruit. Adafruit feather m0 with rfm95 lora radio 900mhz [radiofruit] id: $3178-\$ 34.95$ : Adafruit industries, unique \& fun diy electronics and kits. [Online]. Available: https://www.adafruit.com/product $/ 3178$

[62] A. Del Campo, E. Gambi, L. Montanini, D. Perla, L. Raffaeli, an S. Spinsante, "Mqtt in aal systems for home monitoring of people with dementia," in Personal, Indoor, and Mobile Radio Communication
(PIMRC), 2016 IEEE 27th Annual International Symposium on. IEEE, 2016, pp. 1-6.

[63] B. C. Fargas and M. N. Petersen, "Gps-free geolocation using lora in low-power wans," in Global Internet of Things Summit (GIoTS), 2017. IEEE, 2017, pp. 1-6.

[64] W. Liu, B. Ploderer, and T. Hoang, "In bed with technology: challenges and opportunities for sleep tracking," in Proceedings of the Annual Meeting of the Australian Special Interest Group for Computer Human Interaction. ACM, 2015, pp. 142-151.

[65] S. Spinsante, G. Ciattaglia, A. D. Campo, D. Perla, D. Pigini, G. Cancellieri, and E. Gambi, "A lora enabled building automation architecture based on mqtt," in AEIT International Annual Conference, September 20-22, 2017, Cagliari, Italy, 2017.

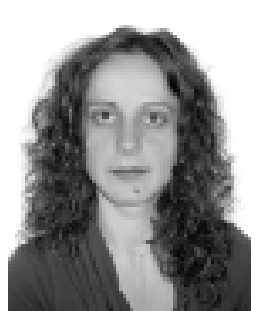

Elma Zanaj received her Laurea Degree in Electronic Engineering, at the Polytechnic University of Tirana, Albania on July 2003. She had finished her $\mathrm{PhD}$ in Electronics, Informatics and Telecommunications Engineering, at DEIT, Università Politecnica delle Marche, Ancona, Italy, December 2008. She has been working at Polytechnic University of Tirana since 2003, and in 2013 she become Associate Professor. She has authored or coauthored over 50 papers in international journals and conferences. Her main research interests include routing optimization and energy efficiency in wireless sensor networks.

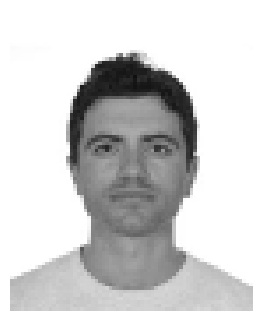

Deivis Disha got the MSc in Electronics Engineering at Polytechnic University of Tirana, Albania in 2011. He worked for 5 years as lector in different universities in Albania. He is currently a Ph.D. Student at the first year at Universita' Politecnica delle Marche in Ancona, Italy. His scientific activity is concerned with data science, artificial intelligence and machine learning with particular interest on the people movement analysis and target characterization based on Automotive Radar applications. In particular, he is now working to the classification of people, or general target, on their characteristic micro-Doppler features.

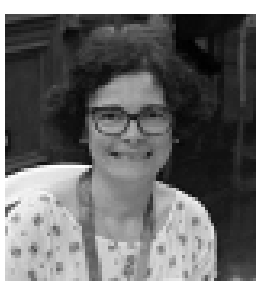

Susanna Spinsante is currently a tenured Assistant Professor in Electrical and Electronic Measurements at the Information Engineering Department (DII) of Università Politecnica delle Marche. She received her PhD in Electronics and Telecommunications Engineering in 2005 from the same University, where she spent several years working in telecommunications and video applications. Now her research interests are focused on the use of wearable sensors to measure physiological parameters and on the evolution of $\mathrm{MV} / \mathrm{LV}$ switchgears towards an IoT approach. She co-authored more than 180 papers in international peer reviewed journals and conference proceedings. She is involved in two European projects, co-funded by AAL JP and MYBL JP, focused on the use of RGBD and wearable sensors for human lifelogging and action understanding.

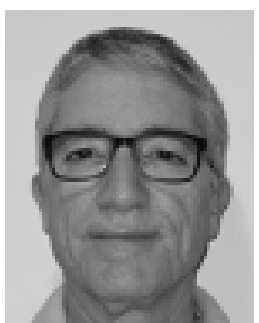

Ennio Gambi received the Electronic Engineering MD at the Universita' Politecnica delle Marche in 1986 and the Master on Microwave Engineering in 1989. Since 1992 he joined the Universita' Politecnica delle Marche in Ancona, where he is, currently, an Associate Professor. Its main research interest is currently focused on the applications of millimeterwave radars, with particular attention to automotivebased radars. At the same time, he is interested on the analysis of radar and video acquisitions of subjects finalized at the extraction of vital parameters, through the implementation of machine learning algorithms. For a long time he has dealt with home automation systems, and on their evolution towards the Environment Assisted Living. 